\title{
Inclusion of Birth and Death Rate in the Modelling of Lassa Fever in Nigeria
}

\author{
O.V Oni ${ }^{*}$, Y.O. Akanle ${ }^{2}$, Ibrahim-Tiamiyu $S^{3}$ \\ ${ }^{1,2,3}$ Department of Statistics, Federal College of Animal Health and Production Technology, Ibadan \\ *Corresponding Author: oniolarewaju@gmail.com
}

Available online at: www.isroset.org

Accepted 06/Aug/2018, Online 30/Aug/2018

\begin{abstract}
The spread dynamics of Lassa fever with inclusion of birth and death rate using the modified Susceptible, Exposed, Infected and Removed model, which is a system of ordinary differential equation. We study the model with demographic effects in Nigeria in order to analyze the stabilities with birth and death rate inclusion; the spread and control of the disease with the use of immunization. Our study reveals that immunization is a very efficient factor in reducing the number of infected individuals in a short time period, early diagnostic of infected humans is the best approach against the spread of the disease.
\end{abstract}

Keywords: Lassa fever, Model, Vaccination, Birth rate, Death rate.

\section{INTRODUCTION}

Lassa fever is an acute viral hemorrhagic fever illness that is known to be endemic in various West African countries including Nigeria. A viral disease majorly caused by Lassa virus. According to the Centers for disease control and prevention (2014), it was first discovered in 1961, in the Lassa town of Borno State, Nigeria. Later on, an endemic situation of Lassa virus was reported in some cities of West African countries of Sierra Leone, Liberia and Guinea, Ogbu (2007). As of 9 June 2017, the outbreak is still active in nine states (Anambra, Bauchi, Cross-River, Edo, Taraba, Nasarawa, Ondo, Plateau, and Kano) in Nigeria. WHO, (2017). 'Since the onset of the 2018 outbreak of Lassa fever in Nigeria, there have been 110 deaths, 78 in positive confirmed cases, 8 in probable cases and 24 in negative cases, according to NCDC latest report. In 2009, the first case from Mali was reported in a traveler living in southern Mali; Ghana reported its first case in late 2011. Isolated cases have also been reported in Côte d'Ivoire and Burkina Faso and there is serologic evidence of Lassa virus infection in Togo and Benin. The number of Lassa virus infections per year in West Africa is estimated at 100,000 to 300,000, with approximately 5,000 deaths. Unfortunately, such estimates are crude, because surveillance for cases of the disease is not uniformly performed. In some areas of Sierra Leone and Liberia, it is known that 10\%-16\% of people admitted to hospitals every year have Lassa fever, which indicates the serious impact of the disease on the population of this region.

The transferor of Lassa Virus is a small rodent (rat), the Multimammate rat of the genus Mastomys. The spread occurs when an individual comes in contact directly with the blood, urine, feaces of rats or other body secretions of an infected person Bawa. M. et al. (2013) \& Gunter S. et al. (2001). Transmission of Lassa virus to humans occurs most commonly through ingestion or inhalation. Mastomys rodents persistently are infected by this virus and in lieu, shed the virus in their urine and droppings. Direct contact with these materials, through touching soiled objects, eating contaminated food, or exposure to open cuts or sores, can lead to infection. Because Mastomys rodents often live in and around homes and scavenge on leftover human food items or poorly stored food, direct contact transmission is common. Mastomys rodents are sometimes consumed as a food source and infection may occur when rodents are caught and prepared. These rodents' lives in the same environment as human, and transmission is by direct contact, and when an individual's breaths in particles in the air containing Lassa virus from an infected person. This aerosol transmission may occur during cleaning activities, such as sweeping, direct contact with infected rodents is not the only way in which people are infected; person-to-person transmission may occur after exposure to virus in the blood, tissue, secretions, or excretions of a Lassa virus-infected individual. The symptoms of Lassa fever begin to show in an individual after being infected between one and three weeks. The disease is mild or has no observable symptoms in up to $80 \%$ of people infected, but $20 \%$ develop a severe multisystem diseases including facial, muscle fatigue, vomiting, cough, 
meningitis and hypertension. Center for diseases control and prevention (2004). The presence of Lassa virus may result into neurological problems including loss of hearing, which may be transient or permanent, tremors and encephalitis.

\section{METHODOLOGY}

Mathematics modeling used for epidemics majorly is the SIR, SIS, and SEIR. In this paper, we try to consider the mathematical method that is closest to real life situation of all, which is the SEIR model that is developed form the SIR mathematical model for epidemic diseases. Since this paper considers, vaccination for the Lassa virus, there was a need to develop the new SEIR model. By means of the SIR and SIS models along with the SEIR and SEIRS models, these models all presumed that the disease spreads in a closed situation. For such models, the population $\mathrm{N}$ is always a constant value since the models do not integrate any births or deaths. In order to advance and implement more lifelike mathematical models close to reality, by assuming a birth rate $\mathrm{b}$ and death rate $\mathrm{d}$, the SEIR model now have a time-varying population $\mathrm{N}(\mathrm{t})$ that is more appropriate in modeling the spread of the Lassa fever virus. Basically, the total population increases by birth at a rate $\mathrm{b}$ and decreases at a rate of d, from the different group of susceptible (S) group, exposed (E) group, infected (I) group, and recovered (R) group, the total population $\mathrm{N}(\mathrm{t})$ adheres to the conservation law, as the sum of the populations of the different compartments that they all vary as a function of time.

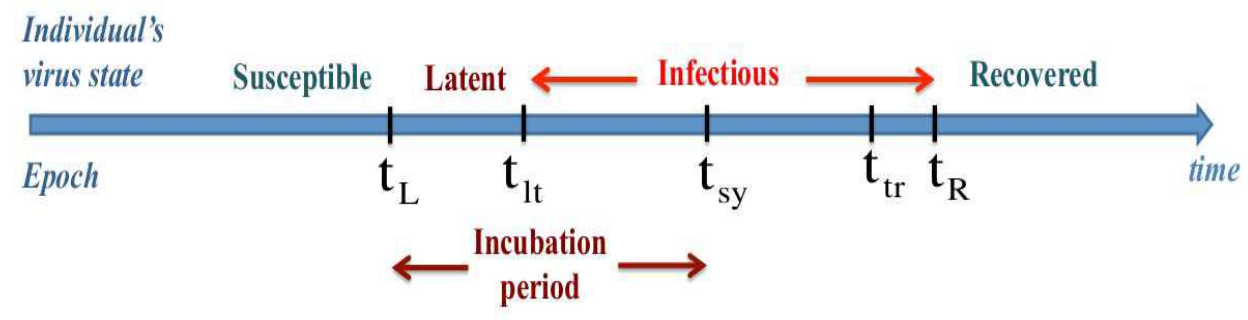

Figure 1: Lassa fever virus progress in an individual by using the SEIR model

The transmission of the virus is described by the following system of nonlinear ordinary differential equations:

$$
\begin{aligned}
& \frac{d S(t)}{d t}=-\beta S(t) I(t) \\
& \frac{d E(t)}{d t}=\beta S(t) I(t)-\gamma E(t) \\
& \frac{d I(t)}{d t}=\gamma E(t)-\mu I(t) \\
& \frac{d R(t)}{d t}=\mu I(t)
\end{aligned}
$$

where $\quad \beta \geq 0$ is the transmission rate; $\gamma \geq 0$ is the infectious rate; and $\mu \geq 0$ is the recovery rate.

The initial conditions are given:

$S(0)=S_{0}>0, E(0)=E_{0} \geq 0, \quad I(0)=I_{0}>0, \quad R(0)=0$.

from (1) $\quad{ }_{d t}^{d}(S(t)+E(t)=I(t)+R(t))=0$, that is, the population $N$ is constant along time: $S(t)+E(t)+I(t)+$ $R(t)=N \quad$ for any $t \geq 0$.

This paper considers the epidemiological model, local stability and the experimental methodology. In the occurrence of an infectious disease such as Lassa fever, the idyllic goal is to either exterminate the virus through preventive measures or launch a mass vaccination program. As an addition to the SEIR with birth and death rate, the Lassa virus vaccinations are administered to newborns (i.e., babies) and non-newborns (i.e., children and adults). For mass immunization programs, newborns or susceptible individuals receive the vaccines and advance to the recovered $\mathrm{R}$ group for the time frame for which the vaccine is effective, they then move back to the susceptible $S$ group to be revaccinated, or otherwise retain being in the recovered $\mathrm{R}$ group if the vaccine administered is for life. By providing the proper Lassa fever vaccines to the public, the mass immunization program serves to reduce the basic reproduction value $\mathrm{R} 0$ to less than unity $(\mathrm{R} 0 \leq 1)$, which causes the infectious disease to die out. For $\mathrm{R} 0$ greater than unity $(\mathrm{R} 0 \geq 1)$, the infective disease does not die out, but causes the occurrence of an epidemic. Primarily, the new SEIR model is a more advanced generalization of the previous models and it incorporates vital dynamics with unequal birth and death rates, vaccinations for both newborns and non-newborns, and temporary immunity for describing the spread of infectious diseases. This model was rescaled using the total time-varying population and analyzed to determine its equilibrium points and corresponding local stabilities of the equilibrium points. In order to test the SEIR model, numerical simulations are run involving a set of arbitrarily defined parameters for horizontal transmission of the infectious disease in the new SEIRS model. 


\subsection{SEIR model with demographic effects}

We study a model with demographic effects that is vital dynamics by considering the birth and death rates, such model is new in the Lassa context. We expand the SEIR model by including demographic effects: we assume a constant birth rate $\delta$ and a natural death rate $\lambda$, obtaining

$$
\begin{gathered}
\frac{d S(t)}{d t}=\delta N-\beta S(t) I(t)-\lambda S(t) \\
\frac{d E(t)}{d t}=\beta S(t) I(t)-\gamma E(t)-\lambda E(t) \\
\frac{d I(t)}{d t}=\gamma E(t)-\mu I(t)-\lambda I(t) \\
\frac{d R(t)}{d t}=\mu I(t)-\lambda R(t)
\end{gathered}
$$

\subsection{Epidemiological Model}

In epidemiology, the new SEIR model (with birth and death rates), vaccinations (newborns and non-newborns), and temporary immunity offers a mathematical description of how the Lassa fever virus spreads in any given population.

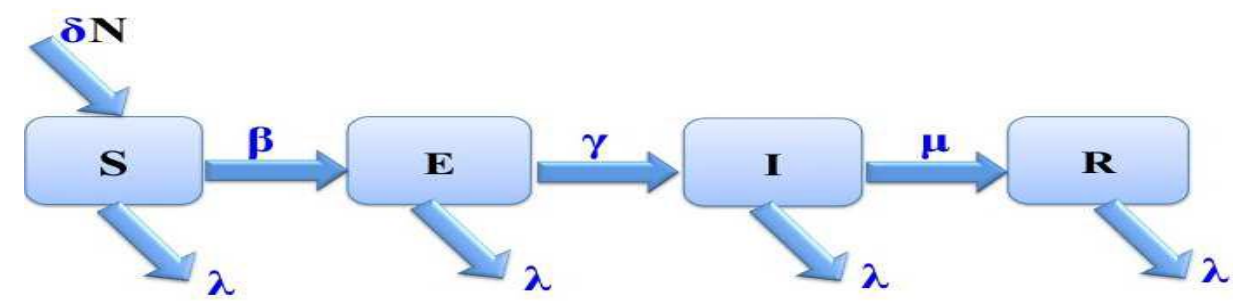

Figure 2: Diagram of the SEIRS Model with Vital dynamics and Vaccination

The table 2 above gives a diagrammatic flow of the SEIRS model, people in a given population from one group to another consisting of Susceptible (S), Exposed (E), Infected (I), and Recovered (R) and then back to being susceptible after some time. For the SEIRS model, the model allows for unequal birth and death rates, vaccinations of both newborns and non-newborns, and temporary immunity from the Lassa fever virus. The nature of the population is that , the initial people in the population before the inception of the Lassa fever are all in the susceptible group (all new born, non- new born and adults), because they have not being vaccinated. Afterwards the vaccination is introduced into the population and the population of the vaccinated people are no longer susceptible but move to the group recovered, while the no vaccinated population of the susceptible group moves to the exposed group after being in contact with the Lassa virus, which last between 6-24 days for the virus to be fully blown and they migrate to the infected group, if not quickly attended to due to the lethal nature of the Lassa virus they are moved to the recovered/ removed group (i.e. death for removed) and cure from the fever (i.e. recovered) who then enjoys a temporary immunity before moving back to the susceptible group if not vaccinated or retains being in the recovered group due to vaccination.

The general description of the model parameters are: birth rate $=\boldsymbol{b}$, death rate $=\boldsymbol{d}$, vaccination rate of new born $=\boldsymbol{v}$, transmission rate of susceptible $=\boldsymbol{\rho}$, the transmission rate of recovered to susceptible $=\boldsymbol{\alpha}$, transmission rate of susceptible to infected $=\boldsymbol{\beta}$, transmission rate of exposed to infected $=\boldsymbol{\sigma}$, transmission rate of infected to recover $=\gamma$, The mean susceptible period $=\frac{1}{\rho}$, temporary mean immune period $=\frac{1}{\alpha}$, mean susceptible period for pre exposed individuals $=\frac{1}{\beta}$, mean latency period $=\frac{1}{\sigma}$, mean infectious period $=\frac{1}{\gamma}$.

Mathematically, the SEIRS model is expressed as a system of ordinary differential equations given by:

$$
\begin{array}{rlrl}
\frac{d s}{d t} & = & b(1-\mathrm{v}) \mathrm{N}-\beta \frac{S I}{N}-d \mathrm{~S}+\alpha \mathrm{R}-\mathrm{Ps} & 1 \\
\frac{d E}{d t} & = & \beta \frac{S I}{N}-\sigma E-d & 2 \\
\frac{d I}{d t} & = & \sigma \mathrm{E}-\gamma \mathrm{I}-d \mathrm{I} & 3 \\
\frac{d R}{d t} & = & b v \mathrm{~N}+\gamma \mathrm{I}-\mathrm{dR}-\mathrm{Ar}+\rho \mathrm{S} & 4
\end{array}
$$


The population is given as:

$$
\begin{array}{rlr}
\mathrm{N}(\mathrm{t})=\mathrm{S}(\mathrm{t})+\mathrm{E}(\mathrm{t})+\mathrm{I}(\mathrm{t})+\mathrm{R}(\mathrm{t}) & 5 \\
\mathrm{~N}^{\prime}(\mathrm{t})=\frac{d N}{d t}=\mathrm{S}^{\prime}(\mathrm{t})+\mathrm{E}^{\prime}(\mathrm{t})+\mathrm{I}^{\prime}(\mathrm{t})+\mathrm{R}^{\prime}(\mathrm{t}) & 6
\end{array}
$$

the rate at which the susceptible gests infected by Lassa is $\beta \frac{S I}{N}$ by substituting equation 4 into $1-3$, the population $\mathrm{N}$ now becomes

$$
\begin{array}{lll}
\frac{S N}{d t}=(b-d) \mathrm{N} & 7
\end{array}
$$

We compute the vale $\mathrm{N}$ by computing the variables

$$
\begin{aligned}
\frac{1}{N} \mathrm{dN} & =(b-d) \mathrm{dt} \\
& =\int \frac{1}{N} \mathrm{dN}=(b-d) \int \mathrm{dt} \\
& =\ln (\mathrm{N})=(b-d) \mathrm{t}
\end{aligned}
$$

Taking the exponential of the population $\mathrm{N}$ in equation 10
$\mathrm{N}=e^{(b-d) \mathrm{t}}$
11

with time-varying population $\mathrm{N}(\mathrm{t})$.Instead of solving the ordinary differential equation system in equations 1 - 3 with known population $\mathrm{N}$ from (8), the transformations: $\mathrm{S}=\frac{S}{N}, \mathrm{e}=\frac{E}{N}, \mathrm{i}=\frac{I}{N}, \mathrm{r}=\frac{R}{N}$, as equations 12-15 respectively, are the number of fractions of people in each group $\mathrm{N}$.

$$
\begin{array}{rlrl}
\frac{d s}{d t} & = & b(1-\mathrm{v}) \mathrm{N}-\beta \mathrm{si}+\alpha \mathrm{r}-(\mathrm{b}+\rho) \mathrm{s} & 12 \\
\frac{d e}{d t} & =\quad \beta \mathrm{si}-(\mathrm{b}+\sigma) \mathrm{e} & 13 \\
\frac{d I}{d t} & =\sigma \mathrm{e}-(\mathrm{b}+\gamma) \mathrm{i}-d \mathrm{I} & 14 \\
\frac{d R}{d t} & =\quad \mathrm{bv}+\gamma \mathrm{i}+\rho \mathrm{s}-(\mathrm{b}+\alpha) \mathrm{r} &
\end{array}
$$

which are all equal to equations. By substitution and transformation equations $12-15$, and equation 5 becomes

$$
\begin{array}{lr}
\mathrm{s}+\mathrm{e}+\mathrm{r}+\mathrm{I}=1 & 16 \\
\mathrm{i}=1-\mathrm{s}-\mathrm{e}-\mathrm{r} & 17 \\
\text { or } & \\
\mathrm{s}^{\prime}+\mathrm{e}^{\prime}+\mathrm{r}^{\prime}+\mathrm{i}^{\prime}=0 & 18
\end{array}
$$

Substituting 18 into12 - 15 and eliminating $\mathrm{r}$, we derive the simplified subsystem:

$$
\begin{array}{rlrl}
\frac{d s}{d t} & = & \alpha+b(1-\mathrm{v})-\alpha \mathrm{e}-\alpha \mathrm{i}-\beta \mathrm{si}-(\mathrm{b}+\rho+\alpha) \mathrm{s} & 19 \\
\frac{d e}{d t} & = & \beta \mathrm{si}-(\mathrm{b}+\sigma) \mathrm{e} & 20 \\
\frac{d I}{d t} & = & \sigma \mathrm{e}-(\mathrm{b}+\gamma) \mathrm{i}-d \mathrm{I} & 21
\end{array}
$$

or

$\frac{d s}{d t}=\mathrm{A}-\alpha \mathrm{e}+-\alpha \mathrm{i}-\beta \mathrm{si}-\mathrm{Bs}$ 


$$
\begin{aligned}
& \frac{d e}{d t}=\quad \beta s \mathrm{si}-\mathrm{Ce} \\
& \frac{d I}{d t}=\sigma \mathrm{e}-\mathrm{Di}
\end{aligned}
$$

With the following positive constrains

$$
\begin{aligned}
& \mathrm{A}=\alpha+b(1-\mathrm{v}), \quad \mathrm{B}=\mathrm{b}+\rho+\alpha, \quad \mathrm{C}=\mathrm{b}+\sigma, \mathrm{D}=\mathrm{b}+\gamma, \text { using s, e, i to solve for } \mathrm{r} \text { in equations } 22- \\
& 24 \\
& \frac{d r}{d t}=b v+\gamma i+\rho s-F r \\
& \text { where } \mathrm{F}=\mathrm{b}+\alpha \quad \text { is a positive constant. }
\end{aligned}
$$

\subsection{Local Stability}

From the transformed subsystem in (18), the local stability is used to determine the disease-free equilibrium (DFE):

$\overline{\mathrm{XDFE}}=(\mathrm{s}, \mathrm{e}, \mathrm{i})=\left(\mathrm{s}^{*}, 0,0\right)$, the endemic equilibrium $(\mathrm{EE})$ is given by:

$\bar{X} \mathrm{EE} \quad=(\mathrm{s}, \mathrm{e}, \mathrm{i})=\left(\mathrm{s}^{*}, \mathrm{e}^{*}, \mathrm{i}^{*}\right)$

The equilibrium points are computed as follows $\frac{d s}{d t}=0, \frac{d e}{d t}=0, \frac{d i}{d t}=0$ for all $\mathrm{s}, \mathrm{e}, \mathrm{i}$ in equations $22-24$

$$
\left[\begin{array}{l}
G \\
H \\
K
\end{array}\right]=\left[\begin{array}{l}
\frac{d s}{d t} \\
\frac{d e}{d t} \\
\frac{d i}{d t}
\end{array}\right]=\left[\begin{array}{ccc}
A-\alpha e & -\alpha i-\beta s i & -B \\
& B s i-C e & \\
\sigma e-D i &
\end{array}\right]
$$

$\mathrm{J}(\mathrm{X})=\mathrm{J}(\mathrm{s}, \mathrm{e}, \mathrm{i})$

$$
=\left[\begin{array}{lll}
\frac{\partial G}{\partial s} & \frac{\partial G}{\partial e} & \frac{\partial G}{\partial i} \\
\frac{\partial H}{\partial s} & \frac{\partial H}{\partial e} & \frac{\partial H}{\partial i} \\
\frac{\partial K}{\partial s} & \frac{\partial K}{\partial e} & \frac{\partial K}{\partial i}
\end{array}\right]=\left[\begin{array}{ccc}
-\beta i-B & -\alpha & -\alpha \beta \\
\beta i & -C & \beta s \\
0 & \sigma & -D
\end{array}\right]
$$

The local stability is derived from the Eigen values $\lambda$,

$|\mathrm{J}(\mathrm{X})-\lambda \mathrm{I}|=0$

\subsection{Disease Free Equilibrium.}

Substituting equation 25 into $\frac{d s}{d t}=0$ of the transformed subsystem of equations $21-25$

The DFE of $\bar{X}$ DFE is computed as

$\frac{d s}{d t}\left|\bar{X}_{\mathrm{DFE}}=0=(\mathrm{A}-\alpha e-\alpha i-\beta s i-B s)\right| \bar{X}_{\mathrm{DFE}}$

it does generate $\mathrm{S}=\frac{A}{B}$ or

$\overline{\mathrm{XDFE}} \quad=(\mathrm{s}, \mathrm{e}, \mathrm{i})=\left(\mathrm{s}^{*}, 0,0\right)=\left(\frac{A}{B}, 0,0\right)$

We take the Jacobian matrix $\mathrm{J}(\mathrm{X})$ at the DFE of $\mathrm{XDFE}=\left(\frac{A}{B}, 0,0\right)$ 
$\mathrm{J}(\mathrm{X})=\left[\begin{array}{ccc}-\beta & -\alpha & -\alpha \beta \frac{A}{B} \\ 0 & -C & \beta \frac{A}{B} \\ 0 & \sigma & -D\end{array}\right]$

The egien values of $\lambda,|J X D F E-\lambda I|=0$

as $=\left[\begin{array}{ccc}-\beta-\lambda & -\alpha & -\alpha \beta \frac{A}{B} \\ 0 & -C-\lambda & \beta \frac{A}{B} \\ 0 & \sigma & -D-\lambda\end{array}\right]=0$

which can be expanded into (-B - $\lambda$ )

$(-\mathrm{B}-\lambda)\left[\begin{array}{cc}-c-\lambda & \beta \frac{A}{B} \\ \sigma & -D-\lambda\end{array}\right]+\left[\begin{array}{cc}0 & \beta \frac{A}{B} \\ 0 & -D-\lambda\end{array}\right]+\left(-\alpha-\beta \frac{A}{B}\right)\left[\begin{array}{cc}0 & -0 \lambda \\ 0 & \sigma\end{array}\right]=0$

Finding the determinants, the Eigen values $\lambda$, is determined by cubic polynomial

$\mathrm{P}(\boldsymbol{\lambda})=\boldsymbol{\lambda}^{3}+(\mathrm{B}+\mathrm{C}+\mathrm{D}) \boldsymbol{\lambda}^{3}+\left(\mathrm{BC}+\mathrm{BD}+\mathrm{CD}-\sigma \beta \frac{A}{B}\right)+(\mathrm{BCD}-\sigma \beta A)=0$

The Eigen values $\lambda$ are dependent on $\sigma, \beta$ and the constants A, B, C and D. By calculating the determinants, the Eigen values by the cubic polynomial.

Routh Hurwitz criteria will be the applied to the cubic polynomial in equation 37 with the co efficient: $a_{1}=B+C+D, \quad a_{2}$ $=\mathrm{BC}+\mathrm{BD}+\mathrm{CD}-\sigma \beta \frac{A}{B}, \mathrm{a}_{3}=\mathrm{BCD}-\sigma \beta A$

By the Routh - Hurwitz criteria for cubic polynomial $\mathrm{P}(\boldsymbol{\lambda})$.

Given that

$$
\begin{array}{cc}
a_{1}>0 & 37 b \text { (i) } \\
a_{3}>0 & 37 b \text { (ii) } \\
a_{1} a_{2}>a_{3} & 37 b \text { (iii) }
\end{array}
$$

all must satisfy XDFE in equation 25 to be locally stable. For the conditions

$1^{\text {st }}$ condition: $\mathrm{a} 1=\mathrm{B}+\mathrm{C}+\mathrm{C}+\mathrm{D}>0$,

since all the constants $\mathrm{B}>0, \mathrm{C}>0$ and $\mathrm{D}>0$ in equation 20, 22.

$2^{\text {nd }}$ condition: $\mathrm{a} 3=\mathrm{BCD}-\sigma \beta A>0, \quad$ if $\frac{B C D}{\sigma \beta A}>1$.

$3^{\text {rd }}$ condition: $\mathrm{a} 1 \mathrm{a} 2=(\mathrm{B}+\mathrm{C}+\mathrm{D})\left(\mathrm{BC}+\mathrm{BD}+\mathrm{CD}-\sigma \beta \frac{A}{B}\right)$

Or

$$
\begin{aligned}
\mathrm{a}_{1} \mathrm{a}_{2}= & \mathrm{B}^{2} \mathrm{C}+\mathrm{B}^{2} \mathrm{D}+3 \mathrm{BCD}+\mathrm{BC}^{2}+\mathrm{C}^{2} \mathrm{D}+\mathrm{BD}^{2}-\mathrm{CD}^{2}-\sigma \beta \frac{A}{B}-\sigma \beta \frac{A C}{B}-\sigma \beta \frac{A D}{B} \\
& =\mathrm{B}^{2} \mathrm{C}+\mathrm{B}^{2} \mathrm{D}+2 \mathrm{BCD}+\mathrm{BC}^{2}+\mathrm{C}^{2} \mathrm{D}+\mathrm{BD}^{2}-\mathrm{CD}^{2}-\sigma \beta \frac{A C}{B}-\sigma \beta \frac{A D}{B}>0
\end{aligned}
$$

Dividing thru by $\mathrm{BC}$

$$
=\frac{B^{2}}{D}+\frac{B^{2}}{C}+2 \mathrm{~B}+\frac{B C}{D}+\frac{B D}{C}+1(\mathrm{CD})>\frac{\sigma \beta A}{B C D}(\mathrm{C}+\mathrm{D})
$$

Where,

$$
\frac{\sigma \beta A}{B C D} 1
$$


With the Routh - Hurrtz all the Eigen values $\boldsymbol{\lambda}$ and cubic polynomial $\mathrm{p}(\boldsymbol{\lambda})$ in equation 31 has negative real parts.

\subsection{Endemic Equilibrium}

From equation 18 the EE $\bar{X} E E$ in 24 is computed by developing a relationship between I and e thru

$\frac{d i}{d t}=0$ as $\frac{d i}{d t}=0=\sigma \mathrm{e}-\mathrm{Di}$

or

$i=\frac{\sigma}{D} \mathrm{e}$

43a ,

where $\frac{e}{i}=\frac{D}{\sigma}$

$43 b$

where $\mathrm{i}=\frac{\sigma}{D} \mathrm{e}$

$43 \mathrm{c}$

By eliminating $\frac{d e}{d t}=0=\beta \mathrm{si}-\mathrm{Ce}$

Where $\mathrm{s}=\frac{C D}{\beta i}$

When 43 is substituted into 45, it gives EE $\bar{X} \mathrm{EE}$ as the

$1^{\text {st }}$ co - ordinates as $\mathrm{s}=\frac{C D}{\beta i}, \quad$ with $\frac{d s}{d t}=0$

$45 b$

as $\frac{d s}{d t}=0=\mathrm{A}-\alpha e-\alpha i-\beta s i-B s$

$$
\mathrm{S}(\beta \mathrm{i}+\mathrm{B})=\mathrm{A}-\alpha e-\alpha i
$$

Substituting $45 \mathrm{~b}$ into 47

$\frac{C D}{\beta \sigma}(\beta \mathrm{i}+\mathrm{B})=\mathrm{A}-\alpha e-\alpha i$

Collecting like terms

$\mathrm{I}\left(\frac{C D}{\sigma}+\alpha\right)=\mathrm{A}-\alpha e-\frac{B C D}{\beta \sigma}$

Simplifying equation $43 \mathrm{a}$

$\left(\frac{\sigma}{D} e\right)+(\beta \mathrm{i}+\mathrm{B})=\mathrm{A}-\alpha e-\alpha i$

Collecting like terms

$\mathrm{I}\left[\left(\frac{\sigma}{D}\right)\left(\frac{C D}{\sigma}+\alpha\right)+\alpha\right]=\mathrm{A}-\frac{B C D}{\beta \sigma}$

By distributing and combining fraction

$\mathrm{e}\left[C+\frac{\alpha \sigma}{D}+\alpha\right]=\mathrm{A}-\frac{B C D}{\beta \sigma}$

$\mathrm{e}\left[\frac{C D+\alpha \sigma+\alpha D}{D}\right]=\frac{B \sigma A-B C D}{\beta \sigma}$

the $2^{\text {nd }}$ co - ordinates of EE $\bar{X} E E$ is given as

$\mathrm{e}\left(\frac{D}{C D+\alpha \sigma+\alpha D}\right)\left(\frac{B \sigma A-B C D}{\beta \sigma}\right)$

$\mathrm{i}=\left(\frac{B \sigma A-B C D}{\beta(C D+\alpha \sigma+\alpha D)}\right)$

$54 b$

Substituting equation $54 \mathrm{~b}$ into $43 \mathrm{c}$ it gives the $3^{\text {rd }} \mathrm{co}-$ ordinates of the EE $\bar{X} \mathrm{EE}$; with the equations $45 \mathrm{~b}, 54 \mathrm{~b}$, and 56 as the 3rd co - ordinates EE $\bar{X} \mathrm{EE}$ is given by

$\bar{X} \mathrm{EE}=(\mathrm{s}, \mathrm{e}, \mathrm{i})=\left(\mathrm{s}^{*}, \mathrm{e}^{*}, \mathrm{i}^{*}\right)$

$=\left[\frac{C D}{\beta \sigma}\left(\frac{D}{C D+\alpha \sigma+\alpha D}\right)\left(\frac{B \sigma A-B C D}{\beta \sigma}\right),\left(\frac{B \sigma A-B C D}{\beta(C D+\alpha \sigma+\alpha D)}\right)\right]$

Its sensible of $\beta \sigma \mathrm{A}-\mathrm{BCD}>0$

57 
Since all the constants A, B, C and D are parameters of $\alpha, \beta$, and $\sigma$ in equations 69 are all positive. Manipulating equations 70 the epidemic conditions Ro is given by

$\mathrm{Ro}=\frac{\beta \sigma A}{B C D}>$

The epidemic condition $\mathrm{R}_{0}$ in 58 is the basic reproduction value

Theorem 1. Let $S(t), E(t), I(t), R(t)$ be a solution of the SEIR model (2). Then the basic reproduction ratio is given by

$$
R_{0}=\frac{\beta \gamma \delta N}{(\mu+\lambda)(\gamma+\lambda) \lambda}
$$

- If $R_{0}>1$, then the equilibrium $P^{*}=\left(S^{*}, E^{*}, I^{*}, R^{*}\right)$ of the virus is obtained, in agreement with expressions (11)- (14), and the virus is able to invade the population.

- If $R_{0}<1$, then the disease free equilibrium $P_{0}=\left(\begin{array}{c}\delta N \\ \lambda\end{array}, 0,0,0\right)$

of the virus is obtained, which corresponds to the case when the virus dies out (no epidemic) and is the most important quantity to consider for analyzing any epidemiological model. In particular, R0 determines whether an epidemic occurs for infectious diseases since $\mathrm{R}_{0}$ is the average number of secondary infections produced by one infected individual during the mean period of infection in a fully susceptible population. If $\mathrm{R} 0<1$, then, on average, the number of new infections produced by one infected individual over the mean course of the infectious disease is less than unity, which implies the infectious disease dies out eventually. Conversely, if R0 > 1, then, on average, the number of new infections produced by one infected individual is greater than unity, which leads to the persistence of the infectious disease as an epidemic. At the EE $\bar{X} E E$ in 55, the Jacobian matrix $\mathrm{J}(\mathrm{X})$ is given by:

$\mathrm{J}(\bar{X} \mathrm{EE})=\left[\begin{array}{ccc}-\beta i *-B & -\alpha & -\alpha B s * \\ B i * & -C & \beta s * \\ 0 & \sigma & -D\end{array}\right]=0$

Expanding it, we have

$(-\beta i *-B-\lambda)\left|\begin{array}{cc}-C-\lambda & B s * \\ \sigma & -D-\lambda\end{array}\right|+\alpha\left|\begin{array}{cc}\beta i * & -C-\lambda \\ 0 & \sigma\end{array}\right|+\left(-\alpha-\beta \mathrm{i}^{*}\right)\left|\begin{array}{cc}\beta i * & -C-\lambda \\ 0 & \sigma\end{array}\right|=0 \quad 61$

Using the cubic polynomial to determine the eigen values of $\lambda$

$\mathrm{P}(\boldsymbol{\lambda})=\lambda^{3}+(\mathrm{B}+\mathrm{C}+\mathrm{D}+\beta \mathrm{i})+(\mathrm{BC}+\mathrm{Bi})+\left(\mathrm{CD}+\beta \mathrm{Ci}^{*}+\beta \mathrm{Di} \mathrm{i}^{*}-\sigma \beta \mathrm{s}\right) \boldsymbol{\lambda}+\left(\mathrm{BCD}+\beta \mathrm{CD} \mathrm{i}^{*}-\sigma \beta \mathrm{B} \mathrm{s}^{*}\right)=0 \quad 62$

or

$\mathrm{P}(\boldsymbol{\lambda})=\boldsymbol{\lambda}^{3}+\left(\mathrm{B}+\mathrm{C}+\mathrm{D}+\frac{\beta \sigma A-B C D}{C D+\alpha \sigma+\alpha D}\right) \lambda^{2}+\left(\mathrm{BC}+\mathrm{BD}+\mathrm{C}\left(\frac{\beta \sigma A-B C D}{C D+\alpha \sigma+\alpha D}\right)\right)+\mathrm{D}\left(\frac{\beta \sigma A-B C D}{C D+\alpha \sigma+\alpha D}\right)+\left(C D\left(\frac{\beta \sigma A-B C D}{C D+\alpha \sigma+\alpha D}\right)\right)=0$ 63

where the three eigenvalues $\lambda$ are dependent on the parameters $\alpha$ and $\beta$, constants $\mathrm{A}, \mathrm{B}, \mathrm{C}$, and $\mathrm{D}$, and first and third coordinates of EE $\bar{X} E E$, namely $\mathrm{s}^{*}$ and $\mathrm{i}^{*}$ in 55 . In a similar manner to the eigenvalues $\lambda$ for the cubic polynomial in 37 , the eigenvalues $\lambda$ for the cubic polynomial in 63 are even more difficult to compute without any specific values for the parameters $\alpha$ and $\beta$ and constants A, B, C, and D. The Routh-Hurwitz criteria with conditions 37 is again applied to now the cubic polynomial in 62 to determine the parameter and constant independent local stability of the EE $\bar{X} \mathrm{EE}$ in (69) with the coefficients:

$$
\begin{gathered}
\mathrm{a} 1=\mathrm{B}+\mathrm{C}+\mathrm{D}+\frac{\beta \sigma A-B C D}{C D+\alpha \sigma+\alpha D} \\
=\frac{\alpha \sigma \beta+\alpha \mathrm{BD}+\mathrm{C} 2 \mathrm{D}+\alpha \sigma \mathrm{C}+\alpha \mathrm{CD}+C D 2+\alpha \sigma D+\alpha D 2+\beta \sigma A}{C D+\alpha \sigma+\alpha D} \\
\mathrm{a}_{2}=\mathrm{BC}+\mathrm{BD}+\mathrm{C}\left(\frac{\beta \sigma A-B C D}{C D+\alpha \sigma+\alpha D}\right)+\mathrm{D}\left(\frac{\beta \sigma A-B C D}{C D+\alpha \sigma+\alpha D}\right) \\
=\frac{\alpha \sigma \beta \mathrm{C}+\alpha \mathrm{BCD}+\alpha \sigma \mathrm{BD} 2+\alpha \mathrm{BD} 2+\beta \sigma \mathrm{AC}+B \sigma A D}{C D+\alpha \sigma+\alpha D} \\
=\mathrm{CD}\left(\frac{\beta \sigma A-B C D}{C D+\alpha \sigma+\alpha D}\right) \\
=\frac{\beta \sigma A C D-B C 2 D 2}{C D+\alpha \sigma+\alpha D}
\end{gathered}
$$




$$
=\frac{\alpha \sigma \beta+\alpha \mathrm{BD}+\mathrm{C} 2 \mathrm{D}+\alpha \sigma \mathrm{C}+\sigma \mathrm{CD}+C D 2+\alpha \sigma D+\alpha D 2+\beta \sigma A}{C D+\alpha \sigma+\alpha D}>0
$$

since the constants $\mathrm{A}>0, \mathrm{~B}>0, \mathrm{C}>0$, and $\mathrm{D}>0$ and parameters $\alpha>0, \beta>0, \sigma>0$ in $37 \mathrm{~b}$ and with the second condition $37 \mathrm{~b}$ :

$$
\begin{aligned}
\mathrm{a} 3= & \frac{\beta \sigma A C D-B C 2 D 2}{C D+\alpha \sigma+\alpha D} \\
& =\beta \sigma A-B C D \alpha
\end{aligned}
$$

$3^{\text {rd }}$ condition $\quad$ a1a2 $=$

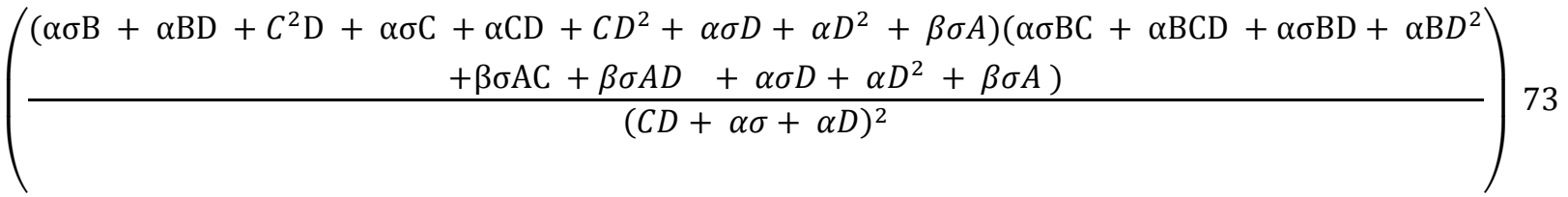

Multiply 70 and 72 by $(C D+\alpha \sigma+\alpha D)^{2}$

Simplifying 37b

$$
\begin{gathered}
\left(\alpha \sigma \mathrm{B}+\alpha \mathrm{BD}+C^{2} \mathrm{D}+\alpha \sigma \mathrm{C}+\alpha \mathrm{CD}+C D^{2}+\alpha \sigma D+\alpha D^{2}+\beta \sigma A\right)\left(\alpha \sigma \mathrm{BC}+\alpha \mathrm{BCD}+\alpha \sigma \mathrm{BD}+\alpha \mathrm{BD} D^{2}+\beta \sigma \mathrm{AC}\right. \\
+\beta \sigma A D)
\end{gathered}
$$

Or

$$
\begin{aligned}
& \alpha^{2} \beta^{2} D^{3}+\alpha^{2} B D^{4}+\alpha^{2} \sigma^{2} B^{2} C+\alpha^{2} \sigma^{2} \beta^{2} D+2 \alpha^{2} \sigma \beta^{2} D^{2}+3 \alpha B C^{2} D^{3}+\alpha^{2} \sigma^{2} B C^{2}+\alpha^{2} \sigma^{2} A C^{3}+ \\
& 2 \alpha^{2} B C D^{3}+\alpha B C D^{4}+\alpha^{2} \sigma^{2} B D^{2}+2 \alpha^{2} \sigma B D^{3}+\beta^{2} \sigma^{2} A D+\alpha^{2} \beta^{2} C D^{3}+\alpha \beta C^{3} D^{2}+\alpha^{2} B C^{2} D^{2}+ \\
& \alpha^{2} \sigma^{2} A B C+2 \alpha^{2} \sigma B^{2} C D+2 \alpha^{2} \sigma \beta^{2} C D+\alpha^{2} \beta^{2} D^{3}+\alpha \sigma B C^{3} D+\alpha \sigma B C^{3} D+3 \alpha \sigma B C^{2} D^{2}+\alpha \sigma A \alpha^{2} \beta^{2} D^{3}+ \\
& \alpha \sigma A C^{3} D+2 \alpha^{2} \sigma^{2} B C D+4 \alpha^{2} \sigma B C D^{2}+2 \alpha^{2} \sigma B^{2} C D^{2}+2 \alpha^{2} \sigma B C^{2} D+\alpha^{2} \sigma A C^{2}+\alpha \sigma B C D^{3}+\alpha \sigma A C^{2} D^{2}+ \\
& \beta \sigma A C D^{5}+\alpha^{2} \sigma^{2} A C D+\alpha \beta \sigma^{2} A D^{2}+\alpha^{2} \sigma A C D^{2}+\alpha^{2} \sigma A C D^{2}+\alpha \beta \sigma A D^{3}+\alpha \sigma^{2} \beta A^{2} C+\alpha \beta \sigma^{2} A B C+ \\
& 2 \alpha \sigma^{2} \beta A B D+\alpha^{2} \sigma A B C D+2 \alpha \beta \sigma A B D^{2}+\alpha \beta \sigma A B C D+B C^{3} D^{3}>0 \quad 75
\end{aligned}
$$

The Routh-Hurwitz criteria, all the eigenvalues $\lambda$ in the cubic polynomial $\mathrm{P}(\boldsymbol{\lambda})$ in 62 have negative real parts to conclude that the DFE XEE in is locally stable with (72).

\section{NUMERICAL Simulation}

We solve numerically the SEIR model with vital dynamics (2) by adopting the parameters presented in the work of Rachah and Torres (2015 and 2016) and Ajala et.al (2017). The early detection of Lassa virus in West Africa is characterized by $\mathrm{R}_{0}=1.95$. The parameters $\beta=0.2, \lambda=0.1887$ and $\mu=0.1$, The initial susceptible, exposed, infectious and recovered populations, are given respectively by $\mathrm{S}(0)=0.88, \mathrm{E}(0)=0.07, \mathrm{I}(0)=0.05, \mathrm{R}(0)=0$. In the numerical resolution of the model, we take the birth rate $\delta=0.412$ and the death rate $\lambda=0.119$, data of population of Nigeria for 2013 are obtained from WHO (2016).

Table 1: Table of parameters and their values

\begin{tabular}{|llll|}
\hline Parameter & Description & Value & Reference \\
\hline Beta & $\begin{array}{l}\text { The parameter controlling how often a susceptible-infected contact } \\
\text { results in a new exposure. }\end{array}$ & 450/25 & ESTIMATED \\
\hline Samma & The rate an infected recovers and moves into the resistant phase. & $1 / 15$ & ESTIMATED \\
\hline Mu & The rate at which an exposed person becomes infective. & $1 / 6$ & ESTIMATED \\
\hline Susceptible & $\begin{array}{l}\text { The natural mortality rate (this is unrelated to disease). This models a } \\
\text { population of a constant size, }\end{array}$ & $\begin{array}{l}\text { The number of susceptible individuals at the beginning of the model } \\
\text { run. }\end{array}$ & ESTIMATED \\
\hline Exposed & The number of exposed individuals at the beginning of the model run. & 0.07 & WHO \\
\hline Infected & The number of infected individuals at the beginning of the model run. & 0.05 & WHO \\
\hline
\end{tabular}


Table 2: Table of constants and their values

\begin{tabular}{|ll|}
\hline Constants & Values \\
\hline A & 0.7140 \\
\hline B & 0.57150 \\
\hline C & 0.33340 \\
\hline D & 0.14290 \\
\hline F & 0.07150 \\
\hline RO & 0.21865 (noepidemic), 3.49840 (epidemic) \\
\hline
\end{tabular}

Table 3: Table of stability

\begin{tabular}{|c|c|c|c|c|c|c|c|}
\hline POINTS & $\mathrm{S}$ & $\mathrm{E}$ & $\mathrm{I}$ & $\lambda 1$ & $\lambda 2$ & $\lambda 3$ & STABILITY \\
\hline DFE & 0.12501 & 0 & 0 & 0.57148 & 0.65737 & $-\mathbf{0 . 1 8 1 0 7}$ & Unstable \\
& & & & & & & \\
\hline EEE & 0.03573 & 0.08928 & 0.208025 & -1.3929 & -0.34731 & $\mathbf{0 . 1 4 0 6 2}$ & stable \\
\hline
\end{tabular}

Table 4: Table of Simulation
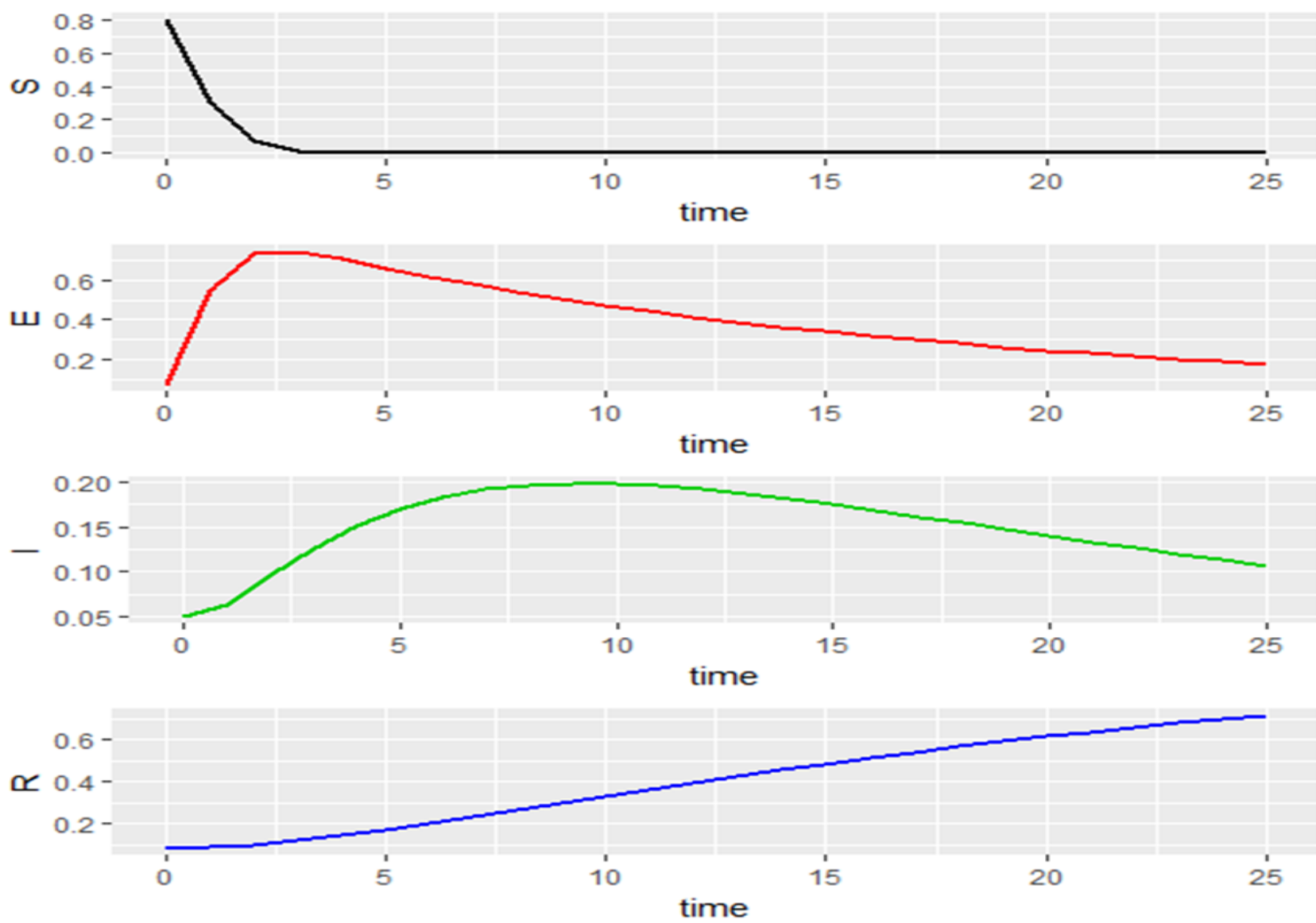

Figure: 3 Simulated result 


\section{CONCLUSION}

We investigated SEIR models in the context of the recent Lassa fever outbreak in Nigeria. Our aim was to study the properties and usefulness of SEIR models with respect to Lassa fever. We began by presenting the basic SEIR model and its mathematical analysis. Then, we added to the model demographic effects in order to analyze the equilibria with vital dynamics. The system of equations of the model was solved numerically. The numerical simulations confirm the theoretical analysis of the equilibria of the model.

\section{REFERENCE}

[1]. Bawa, M., Abdulraham, S., Jimoh, O.R., (2013). Stability Analysis of the Disease Free-Equilibrium State of Lassa fever Disease. Int. J. of Sc.and Math. Edu. 9(2). 115- 123

[2]. Centers for Disease Control and Prevention (2004). Imported Lassa fever. MMWR Morb Mortality Wkly Rep. 53 (38), 894-897.

[3]. Centers for Disease Control and Prevention (2013). Lassa fever fact sheet. MMWR Morb Mortality Wkly Rep.

[4]. Dejen, K. M, and Purnachandra R. K.(2016) An introduction to models for disease dynamics. School of Mathematical \& Statistical Sciences, Hawassa University, Hawassa, Ethiopia

[5]. Gunter, S., Weisner, B., Roth, A.,Gnewing T., Asper,M.,Drosten, C., Emmerich, P.,Peterson, J., Wilczerk, M. Schmitz H.,and Gunther, S. (2001). Lassa fever Encephalotopathy: Lassa virus in Cerebrospinal Fluid but Not in serum. The Journal of Infectious Diseases 184 (3), 345-349. DOI: 10.1086/322033.2001.11443561.

[6]. Marek, B. T (2017), Deterministic Seirs Epidemic Model for Modeling Vital Dynamics, Vaccinations, and Temporary Immunity, Academic Editor: Brigitte Servatius.

[7]. Murali, H. (2009), Spatial Epidemiology. SAMSI.

[8]. Ogbu, O., Ajuluchukwu, E., and Uneke, C. J. (2007). Lassa fever in West Africa Sub-region: an overview. J. Vect. Borne Disease. 44, 111.

[9]. Okuonghae D \& Okuonghae, I. (2006). A mathematical model for Lassa fever. Journal of National Association of Mathematical physics, 10,457-464.

[10]. Oswaldo S. B, Fernando S. M (2016) Dynamic Models in Diseases.

[11]. Ottar, B. (2005), Mathematical Modeling and Simulation Study of SEIR disease and Data Fitting of Ebola Epidemic spreading in West Africa.

[12]. World Health Organization (2016). Lassa fever: Factsheets. Available at http: who.int/mediacenter/factsheets/fs179/en/Accessed on 24/06/2016.

[13]. World Health Organization (2018), WHO Lassa Fever Fact Sheet Available online www.afro.who.int/news/who-moves-contain-Nigeria's Lassa fever outbreak. 